\title{
Non-commutative Zariski geometries and their classical limit
}

\author{
Boris Zilber \\ University of Oxford
}

5 July 2007

\begin{abstract}
We undertake a case study of two series of nonclassical Zariski geometries. We show that these geometries can be realised as representations of certain noncommutative $C^{*}$-algebras and introduce a natural limit construction which for each of the two series produces a classical $U(1)$-gauge field over a 2-dimensional Riemann surface.
\end{abstract}

\section{Introduction}

The notion of a Zariski geometry was introduced in [HZ] as a modeltheoretic generalisation of objects of algebraic geometry and compact complex manifolds.

The main result of paper [HZ] was the classification of non linear (non locally modular) irreducible Zariski geometries of dimension one. The initial hope that every such geometry is definably isomorphic to an algebraic curve over an algebraically closed field $\mathrm{F}$ had to be corrected in the course of the study. The final classification theorem states that given a non linear irreducible 1-dimensional Zariski geometry $M$ there is an algebraically closed field $\mathrm{F}$ definable in $M$ and an algebraic curve $C$ over $\mathrm{F}$ such that $M$ is a finite cover of $C(\mathrm{~F})$, that is there is a Zariski continuous map p : $M \rightarrow C(\mathrm{~F})$ which is a surjection with finite fibres.

The paper [HZ] also provides a class of examples that demonstrates that in general we can not hope to reduce $\mathbf{p}$ to a bijection. Given a smooth algebraic curve $C$ with a big enough group $G$ of regular automorphisms with a nonsplitting finite extension $\tilde{G}$, one can produce a "smooth" irreducible Zariski curve $\tilde{C}$ along with a finite cover $\mathbf{p}: \tilde{C} \rightarrow C$ and $\tilde{G}$ its group of Zariski-definable automorphisms.

Typically $\tilde{C}$ can not be identified with any algebraic curve because $\tilde{G}$ is not embeddable into the group of regular automorphisms of an algebraic curve ([HZ], section 10).

Taking into account known reductions of covers we can say that the above construction of $\tilde{C}$ is essentially the only way to produce a nonclassical Zariski curve. In other words, a general Zariski curve essentially looks like $\tilde{C}$ above. 
A simple example of an unusual group $\tilde{G}$ for such a $\tilde{C}$, used in [HZ], is the class-2-nilpotent group of two generators $\mathbf{u}$ and $\mathbf{v}$ with the central commutator $[\mathbf{u}, \mathbf{v}]$ of finite order $N$. The correspondent $G$ is then the free abelian group on two generators. One can identify this $\tilde{G}$ as the quotient of the integer Heisenberg group $H_{3}(\mathbb{Z})$ by the subgroup of its centre of index $N$.

Also, since the group of regular isomorphisms of the smooth curve $C$ must be infinite, we have very little freedom in choosing $C$; it has to be either the affine line over $\mathrm{F}$, or the torus $\mathrm{F}^{*}$, or an elliptic curve.

This paper undertakes the case study of the geometries of the corresponding $\tilde{C}$ for $C$ an algebraic torus and an affine line.

The most comprehensive modern notion of a geometry is based on the consideration of a coordinate algebra of the geometric object. The classical meaning of a coordinate algebra comes from the algebra of coordinate functions on the object, that is, in our case, functions $\psi: \tilde{C}(\mathrm{~F}) \rightarrow \mathrm{F}$ of a certain class. The most natural algebra of functions seems to be the algebra $\mathrm{F}[\tilde{C}]$ of Zariski continuous (definable) functions. But by the virtue of the construction $\mathrm{F}[\tilde{C}]$ is naturally isomorphic to the $\mathrm{F}[C]$, the algebra of regular functions on the algebraic curve $C$, that is the only geometry which we see by looking into $\mathrm{F}[\tilde{C}]$ is the geometry of the algebraic curve $C$. To see the rest of the structure we had to extend $\mathrm{F}[\tilde{C}]$ by introducing semidefinable functions, which satisfy certain equations but are not uniquely defined by these equations. The F-algebra of $\mathcal{H}(\tilde{C})$ of semi-definable functions contains the necessary information about $\tilde{C}$ but is not canonically defined. On the other hand it is possible to define an F-algebra $\mathcal{A}(\tilde{C})$ of linear operators on $\mathcal{H}(\tilde{C})$ in a canonical way, depending on $\tilde{C}$ only. We proceed with this construction for both examples and write down explicit lists of generators and defining relations for algebras $\mathcal{A}(\tilde{C})$. One particular type of a semi-definable function which we call $*$-functions, of a clearly non-algebraic nature, plays a special role. The $*$-function induces an involution $*$ on $\mathcal{A}$. We show, for $\mathrm{F}=\mathbb{C}$, that $\mathcal{A}$ thus gets the structure of a $C^{*}$-algebra, that is the involution $*$ associates with any $X \in \mathcal{A}$ its formal adjoint operator $X^{*}$ satisfying usual formal requirements. Moreover there is an $\mathcal{A}$-submodule $\mathcal{H}_{+}$of $\mathcal{H}(\tilde{C})$ with an inner product for which $*$ does indeed define adjoint operators.

Our first main theorem states that there is a reverse canonical construction which recovers $\tilde{C}$ from the algebra $\mathcal{A}$ uniquely. The points of $\tilde{C}$ correspond to one-dimensional eigenspaces (states) of certain self-adjoint operators, relations on $C$ correspond to ideals of cartesian powers of a commutative subalgebra of $\mathcal{A}$ and operations $\mathbf{u}$ and $\mathbf{v}$ correspond naturally to actions of certain operators of $\mathcal{A}$ on the states. This scheme is strikingly similar to the operator representations of quantum mechanics. Note that this construction is similar but not identical with the one we used in [Z1].

The final section of the paper concentrates on understanding the limit of the structures $\tilde{C}=\tilde{C}_{N}$, depending on $N$ by the construction of $\tilde{G}$, as $N$ tends to infinity. Amomg many possible ways to define the notion of the limit we found metric considerations most relevant. It turns out possible, when $\mathrm{F}=\mathbb{C}$, to consider metric on each $\tilde{C}_{N}$ and to use correspondingly 
the notion of Hausdorff limit. Our main result in this section states that, for both types of examples, the Hausdorff limit $\tilde{C}_{\infty}$ of $\tilde{C}_{N}$, as $N$ tends to infinity, is the structure identified as the principal $U(1)$-bundle over a Riemann surface with $\mathbf{u}$ and $\mathbf{v}$ defining a connection (covariant derivative) on the bundle. In physicists' terminology this is a gauge field with a nonzero curvature (see e.g. [DFN] or $[\mathrm{S}]$ ).

Combining with the results of the previous section one could speculate that $\tilde{C}_{N}$ are quantum deformations of the classical structure on $\tilde{C}_{\infty}$, and conversely, the latter is the classical limit of the quantum structures.

I am very grateful to Yang-Hui He and Mario Serna for helping me to clarify some of physicists' terminology that I am using in the paper.

\section{Non-algebraic Zariski geometries}

2.0.1 Theorem 1 There exists an irreducible pre-smooth Zariski structure (in particular of dimension 1) which is not interpretable in an algebraically closed field.

The construction

Let $\mathbf{M}=(M, \mathcal{C})$ be an irreducible pre-smooth Zariski structure, $G \leq$ ZAut $M$ (Zariski-continuous bijections) acting freely on $M$ and for some $\tilde{G}$ with finite $H$ :

$$
1 \rightarrow H \rightarrow \tilde{G} \rightarrow^{\mathrm{pr}} G \rightarrow 1 .
$$

Consider a set $X \subseteq M$ of representatives of $G$-orbits: for each $a \in M$, $G \cdot a \cap X$ is a singleton.

Consider the formal set

$$
\tilde{M}(\tilde{G})=\tilde{M}=\tilde{G} \times X
$$

and the projection map

$$
\mathbf{p}:(g, x) \mapsto \operatorname{pr}(g) \cdot x .
$$

Consider also, for each $f \in \tilde{G}$ the function

$$
f:(g, x) \mapsto(f g, x) .
$$

We thus have obtained the structure

$$
\tilde{\mathbf{M}}=\left(\tilde{M},\{f\}_{f \in \tilde{G}} \cup \mathbf{p}^{-1}(\mathcal{C})\right)
$$

on the set $\tilde{M}$ with relations induced from $\mathbf{M}$ together with maps $\{f\}_{f \in \tilde{G}}$. We set the closed subsets of $\tilde{M}^{n}$ to be exactly those which are definable by positive quantifier-free formulas with parameters. Obviously, the structure $\mathbf{M}$ and the map $\mathbf{p}: \tilde{M} \rightarrow M$ are definable in $\tilde{\mathbf{M}}$. Since, for each $f \in \tilde{G}$,

$$
\forall v \mathbf{p} f(v)=f \mathbf{p}(v)
$$


the image $\mathbf{p}(S)$ of a closed subset $S \subseteq \tilde{M}^{n}$ is closed in $\mathbf{M}$. We define $\operatorname{dim} S:=\operatorname{dim} \mathbf{p}(S)$.

Lemma 1 The isomorphism type of $\tilde{\mathbf{M}}$ is determined by $M$ and $\tilde{G}$ only. The theory of $\tilde{\mathbf{M}}$ has quantifier elimination. $\tilde{\mathbf{M}}$ is an irreducible pre-smooth Zariski structure.

Proof. One can use obvious automorphisms of the structure to prove quantifier elimination. The statement of the claim then follows by checking the definitions. The detailed proof is given in [HZ] Proposition 10.1.

Lemma 2. Suppose $H$ does not split, that is for every proper $G_{0}<\tilde{G}$

$$
G_{0} \cdot H \neq \tilde{G}
$$

Then, every equidimensional Zariski expansion $\tilde{\mathbf{M}}^{\prime}$ of $\tilde{\mathbf{M}}$ is irreducible.

Proof Let $C=\tilde{M}^{\prime}$ be an $|H|$-cover of the variety $M$, so $\operatorname{dim} C=$ $\operatorname{dim} M$ and $C$ has at most $|H|$ distinct irreducible components, say $C_{i}, 1 \leq$ $i \leq n$. For generic $y \in M$ the fiber $\mathbf{p}^{-1}(y)$ intersects every $C_{i}$ (otherwise $\mathbf{p}^{-1}(M)$ is not equal to $\left.C\right)$.

Hence $H$ acts transitively on the set of irreducible components. So, $\tilde{G}$ acts transitively on the set of irreducible components, so the setwise stabiliser $G^{0}$ of $C_{1}$ in $\tilde{G}$ is of index $n$ in $\tilde{G}$ and also $H \cap \tilde{G}^{0}$ is of index $n$ in $H$. Hence,

$$
\tilde{G}=G^{0} \cdot H, \text { with } H \nsubseteq G^{0}
$$

contradicting our assumptions.

Lemma 3. $\tilde{G} \leq$ ZAut $\tilde{\mathbf{M}}$, that is $\tilde{G}$ is a subgroup of the group of Zariski-continuous bijecions of $\tilde{\mathbf{M}}$.

Proof Immediate by construction.

Lemma 4 Suppose $\mathbf{M}$ is a rational or elliptic curve (over an algebraically closed field $\mathrm{F}$ of characteristic zero), $H$ does not split, $\tilde{G}$ is nilpotent and for some big enough integer $\mu$ there is a non-abelian subgroup $G_{0} \leq \tilde{G}$

$$
\left|\tilde{G}: G_{0}\right| \geq \mu .
$$

Then $\tilde{\mathbf{M}}$ is not interpretable in an algebraically closed field.

Proof First we show.

Claim. Without loss of generality we may assume that $\tilde{G}$ is infinite.

Recall that $G$ is a subgroup of the group ZAut $M$ of rational (Zariski) automorphisms of $M$. Every algebraic curve is birationally equivalent to a smooth one, so $G$ embeds into the group of birational transformations of a smooth rational curve or an elliptic curve. Now remember that any birational transformation of a smooth algebraic curve is biregular. If $M$ is rational then the group ZAut $M$ is $\operatorname{PGL}(2, \mathrm{~F})$. Choose a semisimple (diagonal) $s \in \operatorname{PGL}(2, \mathrm{~F})$ be an automorphism of infinite order such that 
$\langle s\rangle \cap G=1$ and $G$ commutes with $s$. Then we can replace $G$ by $G^{\prime}=\langle G, s\rangle$ and $\tilde{G}$ by $\tilde{G}^{\prime}=\langle\tilde{G}, s\rangle$ with the trivial action of $s$ on $H$. One can easily see from the construction that the $\tilde{M}^{\prime}$ corresponding to $\tilde{G}^{\prime}$ is the same as $\tilde{M}$, except for the new definable bijection corresponding to $s$.

We can use the same argument when $M$ is an elliptic curve, in which case the group of automorphisms of the curve is given as a semidirect product of a finitely generated abelian group (complex multiplication) acting on the group on the elliptic curve $E(\mathrm{~F})$.

Now, assuming that $\tilde{M}$ is definable in an algebraically closed field $\mathrm{F}^{\prime}$ we will have that $\mathrm{F}$ is definable in $\mathrm{F}^{\prime}$. It is known to imply that $\mathrm{F}^{\prime}$ is definably isomorphic to $\mathrm{F}$, so we may assume that $\mathrm{F}^{\prime}=\mathrm{F}$.

Also, since $\operatorname{dim} \tilde{M}=\operatorname{dim} M=1$, it follows that $\tilde{M}$ up to finitely many points is in a bijective definable correspondence with a smooth algebraic curve, say $C=C(\mathrm{~F})$.

$\tilde{G}$ then by the argument above is embedded into the group of rational automorphisms of $C$.

The automorphism group is finite if genus of the curve is 2 or higher, so by the Claim we can have only rational or elliptic curve for $C$.

Consider first the case when $C$ is rational. The automorphism group then is $\operatorname{PGL}(2, F)$. Since $\tilde{G}$ is nilpotent its Zariski closure in $\operatorname{PGL}(2, \mathrm{~F})$ is an infinite nilpotent group $U$. Let $U^{0}$ be the connected component of $U$, which is a normal subgroup of finite index. By Malcev's Theorem (see [Merzliakov], 45.1) there is a number $\mu$ (dependent only on the size of the matrix group in question but not on $U$ ) such that some normal subgroup $U^{0}$ of $U$ of index at most $\mu$ is a subgroup of the unipotent group

$$
\left(\begin{array}{ll}
1 & z \\
0 & 1
\end{array}\right)
$$

this is Abelian, contradicting the assumption that $\tilde{G}$ has no abelian subgroups of index less than $\mu$.

In case $C$ is an elliptic curve the group of automorphisms is a semidirect product of a finitely generated abelian group (complex multiplication) acting freely on the abelian group of the elliptic curve. This group has no nilpotent non-abelian subgroups. This finishes the proof of Lemma 4 and of the theorem.

In general it is harder to analyse the situation when $\operatorname{dim} M>1$ since the group of birational automorphisms is not so immediately reducible to the group of biregular automorphisms of a smooth variety in higher dimensions. But nevertheless the same method can prove the useful fact that the construction produces examples essentially of non algebro-geometric nature.

Proposition 1 (i) Suppose $M$ is an abelian variety, $H$ does not split and $\tilde{G}$ is nilpotent not abelian. Then $\tilde{M}$ can not be an algebraic variety with $p: \tilde{M} \rightarrow M$ a regular map. 
(ii) Suppose $M$ is the (semi-abelian) variety $\left(\mathrm{F}^{\times}\right)^{n}$. Suppose also that $\tilde{G}$ is nilpotent and for some big enough integer $\mu=\mu(n)$ has no abelian subgroup $G_{0}$ of index bigger than $\mu$. Then $\tilde{M}$ can not be an algebraic variety with $p: \tilde{M} \rightarrow M$ a regular map.

Proof (i) If $M$ is an abelian variety and $\tilde{M}$ were algebraic, the map $p: \tilde{M} \rightarrow M$ has to be unramified since all its fibers are of the same order (equal to $|H|$ ). Hence $\tilde{M}$ being a finite unramified cover must have the same unversal cover as $M$ has. So, $\tilde{M}$ must be an abelian variety as well. The group of automorphisms of an abelian variety $\mathcal{A}$ without complex multiplication is the abelian group $\mathcal{A}(\mathrm{F})$. The contradiction.

(ii) Same argument as in (i) proves that $\tilde{M}$ has to be isomorphic to $\left(\mathrm{F}^{\times}\right)^{n}$. The Malcev theorem cited above finishes the proof. $\square$

Proposition 2 Suppose $M$ is an F-variety and, in the construction of $\tilde{M}$, the group $\tilde{G}$ is finite. Then $\tilde{M}$ is definable in any expansion of the field $\mathrm{F}$ by a total linear order.

In particular, if $M$ is a complex variety, $\tilde{M}$ is definable in the reals.

Proof Extend the ordering of $\mathrm{F}$ to a linear order of $M$ and define

$$
S:=\{s \in M: s=\min G \cdot s\} .
$$

The rest of the construction of $\tilde{M}$ is definable

Remark In other known examples of non-algebraic $\tilde{M}$ (with $G$ infinite) $\tilde{M}$ is still definable in any expansion of the field $\mathrm{F}$ by a total linear order. In particular, for the example considered in this paper, see section ??.

Problem (i) Classify Zariski structures definable in the reals.

(ii) Classify Zariski structures definable in the reals as a smooth real manifold.

(iii) Find new Zariski structures definable in $\mathbb{R}_{a n}$ as a smooth real manifold.

\section{Examples}

Let $N$ be a positive integer and $\mathrm{F}$ an algebraically closed field of characteristic prime to $N$. Consider the groups given by generators and defining relations,

$$
\begin{gathered}
G=\langle u, v: u v=v u\rangle \\
\tilde{G}=\tilde{G}_{N}=\left\langle\mathbf{u}, \mathbf{v}:[\mathbf{u},[\mathbf{u}, \mathbf{v}]]=[\mathbf{v},[\mathbf{u}, \mathbf{v}]]=1=[\mathbf{u}, \mathbf{v}]^{N}\right\rangle,
\end{gathered}
$$

where $[\mathbf{u}, \mathbf{v}]$ stands for the commutator $\mathbf{v u v} \mathbf{v}^{-1} \mathbf{u}^{-1}$.

We will consider two examples of the construction of a one-dimensional $\tilde{M}$ from an algebraic curve $M$ using the groups $G$ and $\tilde{G}$. By section $2 G$ is going to be a subgroup of the group of rational automorphisms of $M$, so $M$ has to be of genus 0 or 1 . In our examples $M$ is the algebraic torus $\mathrm{F}^{*}$ and the affine line $\mathrm{F}$. 


\subsection{The $N$-cover of the affine line.}

3.1.1 We assume here that the characteristic of $F$ is 0 .

Let $a, b \in \mathrm{F}$ be additively independent.

$G$ acts on $\mathrm{F}$ :

$$
u x=a+x, v x=b+x .
$$

Taking $M$ to be $\mathrm{F}$ this determines, by subsection 2 a presmooth nonalgebraic Zariski curve $\tilde{M}$ which from now on we denote $\mathrm{P}_{N}$, and $P_{N}$ will stand for the universe of this structure.

The correspondent definition for the covering map $p: \tilde{M} \rightarrow M=\mathrm{F}$ then gives us

$$
\mathbf{p}(\mathbf{u} t)=a+\mathbf{p}(t), \quad \mathbf{p}(\mathbf{v} t)=b+\mathbf{p}(t) .
$$

\subsubsection{Semi-definable functions on $\mathrm{P}_{N}$.}

Lemma There are functions $y$ and $z$

$$
P_{N} \rightarrow \mathrm{F}
$$

satisfying the following functional equations, for any $t \in P_{N}$,

$$
\begin{gathered}
\mathbf{y}^{N}(t)=1, \mathbf{y}(\mathbf{u} t)=\epsilon \mathbf{y}(t), \mathbf{y}(\mathbf{v} t)=\mathbf{y}(t) \\
\mathbf{z}^{N}(t)=1, \mathbf{z}(\mathbf{u} t)=\mathbf{z}(t), \mathbf{z}(\mathbf{v} t)=\mathbf{y}(t)^{-1} \cdot \mathbf{z}(t) .
\end{gathered}
$$

Proof Choose a subset $S \subseteq M=\mathrm{F}$ of representatives of $G$-orbits, that is $\mathrm{F}=G+S$. By the construction in section 2 we can identify $P_{N}=\tilde{M}$ with $\tilde{G} \times S$ in such a way that $\mathbf{p}(g, s)=\operatorname{pr}(g)+s$. This means that, for any $s \in S$, a $t$ in $\tilde{G} \cdot s$ is of the form $t=\mathbf{u}^{m} \mathbf{v}^{n}[\mathbf{u}, \mathbf{v}]^{\ell} \cdot s$ and

$$
\mathbf{p}\left(\mathbf{u}^{m} \mathbf{v}^{n}[\mathbf{u}, \mathbf{v}]^{\ell} \cdot s\right):=m a+n b+s .
$$

Set

$$
\begin{aligned}
& \mathbf{y}\left(\mathbf{u}^{m} \mathbf{v}^{n}[\mathbf{u}, \mathbf{v}]^{\ell} \cdot s\right):=\epsilon^{m} \\
& \mathbf{z}\left(\mathbf{u}^{m} \mathbf{v}^{n}[\mathbf{u}, \mathbf{v}]^{\ell} \cdot s\right):=\epsilon^{l} .
\end{aligned}
$$

This satisfies (2) and (3).

Remark. Notice, that it follows from (11)-(3):

1. $\mathbf{p}$ is surjective and $N$-to- 1 , with fibres of the form

$$
\mathbf{p}^{-1}(\lambda)=H t, \quad H=\left\{[\mathbf{u}, \mathbf{v}]^{\ell}: 0 \leq l<N\right\} .
$$

2. $\mathbf{y}([\mathbf{u}, \mathbf{v}] t)=\mathbf{y}(t)$,

3. $\mathbf{z}([\mathbf{u}, \mathbf{v}] t)=\epsilon \mathbf{z}(t)$.

3.1.3 Denote $\mathrm{F}[N]=\left\{\xi \in \mathrm{F}: \xi^{N}=1\right\}$ and define the band function on $\mathrm{F}$ as a function bd: $\mathrm{F} \rightarrow \mathrm{F}[N]$.

Set for $\lambda \in \mathrm{F}$

$$
\operatorname{bd}(\lambda)=y(t), \text { if } \mathbf{p}(t)=\lambda,
$$


This is well-defined by the remark in 3.1 .2

Acting by $\mathbf{u}$ on $t$ and using (11) and (2) we have

$$
\operatorname{bd}(a+\lambda)=\epsilon \mathrm{bd} \lambda .
$$

Acting by $\mathbf{v}$ we obtain

$$
\operatorname{bd}(b+\lambda)=\operatorname{bd} \lambda .
$$

Remark In a more general context we are going to call the band function and the angular function of the next section $*$-functions, explaining the reasons for this at the end of this section.

Proposition The structure $\mathrm{P}_{N}$ is definable in

$$
(\mathrm{F},+, \cdot, \mathrm{bd}) \text {. }
$$

\section{Proof Set}

$$
P_{N}=\mathrm{F} \times \mathrm{F}[N]=\left\{\left\langle x, \epsilon^{\ell}\right\rangle: x \in \mathrm{F}, \ell=0, \ldots, N-1\right\}
$$

and define the maps

$$
\left.\mathbf{p}\left(\left\langle x, \epsilon^{\ell}\right\rangle\right):=x, \mathbf{u}\left(\left\langle x, \epsilon^{\ell}\right\rangle\right):=\left\langle a+x, \epsilon^{\ell}\right\rangle\right), \mathbf{v}\left(\left\langle x, \epsilon^{\ell}\right\rangle\right):=\left\langle b+x, \epsilon^{\ell} \operatorname{bd}(x)\right\rangle .
$$

One checks easily that the action of $\tilde{G}$ is well-defined and that (1) holds.

Remark One can easily define in $(\mathrm{F},+, \cdot, \mathrm{bd})$ functions $\mathbf{x}, \mathbf{y}$ and $\mathbf{z}$ satisfying (2) and (3).

Assuming that $\mathrm{F}=\mathbb{C}$ and for simplicity that $a \in i \mathbb{R}$ and $b \in \mathbb{R}$, both nonzero, we may define, for $z \in \mathbb{C}$,

$$
\operatorname{bd}(z):=\exp \left(\frac{2 \pi i}{N}\left[\operatorname{Re}\left(\frac{z}{a}\right)\right]\right)
$$

This satisfies (4) and (5) and so $\mathrm{P}_{N}$ over $\mathbb{C}$ is definable in $\mathbb{C}$ equipped with the measurable but not continuous function above.

Question Does there exist a supersimple structure of the form $(\mathrm{F},+, \cdot, \mathrm{bd})$ satisfying (4) and (5)?

\subsubsection{The space of semi-definable functions.}

Let $\mathcal{H}$ be an F-algebra containing all the functions $P_{N} \rightarrow \mathrm{F}$ which are definable in $\mathrm{P}_{N}$ expanded by $x, y, z$.

We define linear operators $\mathbf{X}, \mathbf{Y}, \mathbf{Z}, \mathbf{U}$ and $\mathbf{V}$ on $\mathcal{H}$ :

$$
\begin{aligned}
& \mathbf{X}: \psi(t) \mapsto \mathbf{p}(t) \cdot \psi(t), \\
& \mathbf{Y}: \psi(t) \mapsto \mathbf{y}(t) \cdot \psi(t), \\
& \mathbf{Z}: \psi(t) \mapsto \mathbf{z}(t) \cdot \psi(t), \\
& \mathbf{U}: \psi(t) \mapsto \psi(\mathbf{u} t), \\
& \mathbf{V}: \psi(t) \mapsto \psi(\mathbf{v} t) .
\end{aligned}
$$


Denote $\tilde{G}^{*}$ the group generated by the operators $\mathbf{U}, \mathbf{V}, \mathbf{U}^{-1}, \mathbf{V}^{-1}$, denote $\mathfrak{X}_{\epsilon}$ (or simply $\mathfrak{X}$ ) the F-algebra $\mathrm{F}[\mathbf{X}, \mathbf{Y}, \mathbf{Z}]$ and $\mathcal{A}_{\epsilon}$ (or simply $\mathcal{A}$ ) the extension of the $\mathrm{F}$ algebra $\mathfrak{X}_{\epsilon}$ by $\tilde{G}^{*}$.

While elements of $\mathcal{H}$ and $\mathcal{H}$ as a whole are not uniquely defined we prove in 3.1.6 that $\mathcal{A}$ is exactly the algebra of operators on $\mathcal{H}$ generated by $\mathbf{X}, \mathbf{Y}, \mathbf{Z}, \mathbf{U}$ and $\mathbf{V}$ satisfying the following defining relations ( $\mathbf{E}$ stands below for the commutator $[\mathbf{U}, \mathbf{V}])$ :

$$
\begin{aligned}
& \mathbf{X Y}=\mathbf{Y X} ; \mathbf{X Z}=\mathbf{Z X} ; \mathbf{Y Z}=\mathbf{Z Y} \\
& \mathbf{Y}^{N}=1 ; \mathbf{Z}^{N}=1 ; \\
& \mathbf{U X}-\mathbf{X U}=a \mathbf{U} ; \mathbf{V X}-\mathbf{X V}=b \mathbf{V} ; \\
& \mathbf{U Y}=\epsilon \mathbf{Y U} ; \mathbf{Y} \mathbf{V}=\mathbf{V Y} ; \\
& \mathbf{Z U}=\mathbf{U Z} ; \\
& \mathbf{V Z}=\mathbf{Y Z V} ; \\
& \mathbf{U E}=\mathbf{E U} ; \mathbf{V E}=\mathbf{E V} ; \mathbf{E}^{N}=1
\end{aligned}
$$

3.1.5 Let $\operatorname{Max}(\mathfrak{X})$ be the set of isomorphism classes of 1-dimensional irreducible $\mathfrak{X}$-modules.

Lemma $1 \operatorname{Max}(\mathfrak{X})$ can be represented by 1 -dimensional modules $\left\langle e_{\mu, \xi, \zeta}\right\rangle$ ( $e_{\mu, \xi, \zeta}$ generating the module) for $\mu \in \mathrm{F}, \xi, \zeta \in \mathrm{F}[N]$, defined by the action on the generating vector as follows:

$$
\mathbf{X} e_{\mu, \xi, \zeta}=\mu e_{\mu, \xi, \zeta}, \mathbf{Y} e_{\mu, \xi, \zeta}=\xi e_{\mu, \xi, \zeta}, \mathbf{Z} e_{\mu, \xi, \zeta}=\zeta e_{\mu, \xi, \zeta}
$$

Proof This is a standard fact of commutative algebra.

Remark We can find some of the $e_{\mu, \xi, \zeta}$ in $\mathcal{H}$, which by definition contains the following Dirac delta-functions, for any $p \in P_{N}$,

$$
\delta_{p}(t)=\left\{\begin{array}{l}
1, \text { if } t=p \\
0, \text { otherwise }
\end{array}\right.
$$

One checks that

$$
\mathbf{X} \delta_{p}=\mathbf{p}(p) \cdot \delta_{p}, \quad \mathbf{Y} \delta_{p}=\mathbf{y}(p) \cdot \delta_{p}, \quad \mathbf{Z} \delta_{p}=\mathbf{z}(p) \cdot \delta_{p} .
$$

That is we get $e_{\mathbf{p}(p), \mathbf{y}(p), \mathbf{z}(p)}$ in this way.

Assuming $\mathrm{F}$ is endowed with a fixed function bd $: \mathrm{F} \rightarrow \mathrm{F}[N]$ we call $\langle\mu, \xi, \zeta\rangle$ as above real oriented if

$$
\operatorname{bd} \mu=\xi .
$$

Correspondingly, we call the module $\left\langle e_{\mu, \xi, \zeta}\right\rangle$ real oriented if $\langle\mu, \xi, \zeta\rangle$ is.

$\operatorname{Max}^{+}(\mathfrak{X})$ will denote the subspace of $\operatorname{Max}(\mathfrak{X})$ consisting of real oriented modules $\left\langle e_{\mu, \xi, \zeta}\right\rangle$.

Lemma $\mathbf{2}\langle\mu, \xi, \zeta\rangle$ is real oriented if and only if

$$
\langle\mu, \xi, \zeta\rangle=\langle\mathbf{p}(t), \mathbf{y}(t), \mathbf{z}(t)\rangle,
$$


for some $t \in P_{N}$.

Proof It follows from the definition of bd that $\langle\mathbf{p}(t), \mathbf{y}(t), \mathbf{z}(t)\rangle$ is real oriented.

Assume now that $\langle\mu, \xi, \zeta\rangle$ is real oriented. Since $\mathbf{p}$ is a surjection, there is $t^{\prime} \in P_{N}$ such that $\mathbf{p}\left(t^{\prime}\right)=\mu$. By the definition of bd, $\mathbf{y}\left(t^{\prime}\right)=\mathrm{bd} \mu$. By the Remark in 3.1 .2 both values stay the same if we replace $t^{\prime}$ by $t=[\mathbf{u}, \mathbf{v}]^{k} t^{\prime}$. By the same Remark, for some $k, \mathbf{z}(t)=\zeta$.

Now we introduce an infinite-dimensional $\mathcal{A}$-module $\mathcal{H}_{0}$. As a vector space $\mathcal{H}_{0}$ is spanned by $\left\{e_{\mu, \xi, \zeta}: \mu \in \mathrm{F}, \xi, \zeta \in \mathrm{F}[N]\right\}$. The action of the generators of $\mathcal{A}$ on $\mathcal{H}_{0}$ is defined on $e_{\mu, \xi, \zeta}$ in accordance with the defining relations of $\mathcal{A}$. So, since

$$
\begin{gathered}
\mathbf{X} \mathbf{U} e_{\mu, \xi, \zeta}=(\mathbf{U X}-a \mathbf{U}) e_{\mu, \xi, \zeta}=(\mu-a) \mathbf{U} e_{\mu, \xi, \zeta}, \\
\mathbf{Y} \mathbf{U} e_{\mu, \xi, \zeta}=\epsilon^{-1} \mathbf{U} \mathbf{Y} e_{\mu, \xi, \zeta}=\epsilon^{-1} \xi \mathbf{U} e_{\mu, \xi, \zeta}, \\
\mathbf{Z U} e_{\mu, \xi, \zeta}=\mathbf{U Z} e_{\mu, \xi, \zeta}=\zeta \mathbf{U} e_{\mu, \xi, \zeta}
\end{gathered}
$$

and

$$
\begin{gathered}
\mathbf{X V} e_{\mu, \xi, \zeta}=(\mathbf{V X}-b \mathbf{V}) e_{\mu, \xi, \zeta}=(\mu-b) \mathbf{V} e_{\mu, \xi, \zeta}, \\
\mathbf{Y V} e_{\mu, \xi, \zeta}=\mathbf{V} \mathbf{Y} e_{\mu, \xi, \zeta}=\xi \mathbf{U} e_{\mu, \xi, \zeta}, \\
\mathbf{Z V} e_{\mu, \xi, \zeta}=\mathbf{V Y} \mathbf{Y}^{-1} \mathbf{Z} e_{\mu, \xi, \zeta}=\xi^{-1} \zeta \mathbf{V} e_{\mu, \xi, \zeta},
\end{gathered}
$$

we set

$$
\mathbf{U} e_{\mu, \xi, \zeta}:=e_{\mathbf{u}\langle\mu, \xi, \zeta\rangle}, \text { with } \mathbf{u}\langle\mu, \xi, \zeta\rangle=\left\langle\mu-a, \epsilon^{-1} \xi, \zeta\right\rangle
$$

and

$$
\mathbf{V} e_{\mu, \xi, \zeta}:=e_{\mathbf{v}\langle\mu, \xi, \zeta\rangle}, \text { with } \mathbf{v}\langle\mu, \xi, \zeta\rangle=\left\langle\mu-b, \xi, \xi^{-1} \zeta\right\rangle .
$$

From now on we identify $\operatorname{Max}^{+}(\mathfrak{X})$ with the family of real oriented 1-dimensional $\mathfrak{X}$-eigenspaces of $\mathcal{H}_{0}$.

Theorem 2 (i) There is a bijective correspondence $\Xi: \operatorname{Max}^{+}(\mathfrak{X}) \rightarrow P_{N}$ between the set of real oriented $\mathfrak{X}$-eigensubspaces of $\mathcal{H}_{0}$ and $P_{N}$.

(ii) The action of $\tilde{G}^{*}$ on $\mathcal{H}_{0}$ induces an action on $\operatorname{Max}(\mathfrak{X})$ and leaves $\operatorname{Max}^{+}(\mathfrak{X})$ setwise invariant. The correspondence $\Xi$ transfers anti-isomorphically the natural action of $\tilde{G}^{*}$ on $\operatorname{Max}^{+}(\mathfrak{X})$ to a natural action of $\tilde{G}$ on $P_{N}$.

(iii) The map

$$
\mathbf{p}_{\mathfrak{X}}:\left\langle e_{\mu, \xi, \zeta}\right\rangle \mapsto \mu
$$

is a $N$-to-1-surjection $\operatorname{Max}^{+}(\mathfrak{X}) \rightarrow \mathrm{F}$ such that

$$
\left(\operatorname{Max}^{+}(\mathfrak{X}), \mathbf{U}, \mathbf{V}, \mathbf{p}_{\mathfrak{X}}, \mathrm{F}\right) \cong_{\xi}\left(P_{N}, \mathbf{u}, \mathbf{v}, \mathbf{p}, \mathrm{F}\right) .
$$

Proof (i) Immediate by Lemma 2 .

(ii) Indeed, by the definition above the action of $\mathbf{U}$ and $\mathbf{V}$ corresponds to the action on real oriented $N$-tuples:

$\mathbf{U}:\langle\mathbf{p}(t), \mathbf{y}(t), \mathbf{z}(t)\rangle \mapsto\left\langle\mathbf{p}(t)-a, \epsilon^{-1} \mathbf{y}(t), \mathbf{z}(t)\right\rangle=\left\langle\mathbf{p}\left(\mathbf{u}^{-1} t\right), \mathbf{y}\left(\mathbf{u}^{-1} t\right), \mathbf{z}\left(\mathbf{u}^{-1} t\right)\right\rangle$ 


$$
\mathbf{V}:\left\langle\mathbf{p}(t)-b, \mathbf{y}(t), \mathbf{y}(t)^{-1} \mathbf{z}(t)\right\rangle \mapsto\left\langle\mathbf{p}\left(\mathbf{v}^{-1} t\right), \mathbf{y}\left(\mathbf{v}^{-1} t\right), \mathbf{z}\left(\mathbf{v}^{-1} t\right)\right\rangle .
$$

(iii) Immediate from (i) and (ii). $\square$

\subsection{6 $C^{*}$-representation.}

Our aim here is to introduce a natural $C^{*}$-algebra structure on $\mathcal{A}$. In fact we will do it for an extension $\mathcal{A}^{\#}$ of $\mathcal{A}$. recall that a $\mathbb{C}$-algebra $\mathcal{A}$ with a norm is called a $C^{*}$-algebra if there is a map $*: \mathcal{A} \rightarrow \mathcal{A}$ satisfying the following properties: for all $X, Y \in \mathcal{A}$ :

$$
\begin{gathered}
\left(X^{*}\right)^{*}=X, \\
(X Y)^{*}=Y^{*} X^{*}, \\
(X+Y)^{*}=X^{*}+Y^{*},
\end{gathered}
$$

for every $\lambda \in \mathbb{C}$ and every $X \in \mathcal{A}$ :

$$
(\lambda X)^{*}=\bar{\lambda} X^{*}
$$

and

$$
\left\|X^{*} X\right\|=\|X\|^{2} .
$$

In the last condition we do not assume that the norm is always finite.

We will assume $\mathrm{F}=\mathbb{C}, a=\frac{2 \pi i}{N}, b \in \mathbb{R}$ and start by extending the space $\mathcal{H}$ of semi-definable functions with a function $\mathbf{w}: P_{N} \rightarrow \mathbb{C}$ such that

$$
\exp \mathbf{w}=\mathbf{y}, \mathbf{w}(\mathbf{u} t)=\frac{2 \pi i}{N}+\mathbf{w}(t), \mathbf{w}(\mathbf{v} t)=\mathbf{w}(t) .
$$

We can easily do this by setting as in (3.1.2)

$$
\mathbf{w}\left(\mathbf{u}^{m} \mathbf{v}^{n}[\mathbf{u}, \mathbf{v}]^{\ell} \cdot s\right):=\frac{2 \pi i m}{N} .
$$

Now we extend $\mathcal{A}$ to $\mathcal{A}^{\#}$ by adding the new operator

$$
\mathbf{W}: \psi \mapsto \mathbf{w} \psi
$$

which obviously satisfies

$$
\begin{gathered}
\mathbf{W X}=\mathbf{X W}, \mathbf{W} \mathbf{Y}=\mathbf{Y W}, \mathbf{W Z}=\mathbf{Z W} . \\
\mathbf{U W}=\frac{2 \pi i}{N}+\mathbf{W} \mathbf{U}, \mathbf{V W}=\mathbf{W} \mathbf{V} .
\end{gathered}
$$

We set

$$
\begin{gathered}
\mathbf{U}^{*}:=\mathbf{U}^{-1}, \mathbf{V}^{*}:=\mathbf{V}^{-1} \\
\mathbf{Y}^{*}:=\mathbf{Y}^{-1}, \mathbf{W}^{*}:=-\mathbf{W}, \mathbf{X}^{*}:=\mathbf{X}-2 \mathbf{W},
\end{gathered}
$$

implying that $\mathbf{U}, \mathbf{V}$ and $\mathbf{Y}$ are unitary and $i \mathbf{W}$ and $\mathbf{X}-\mathbf{W}$ are formally selfadjoint.

Lemma There is a representation of $\mathcal{A}^{\#}$ in an inner product space such that $\mathbf{U}, \mathbf{V}$ and $\mathbf{Y}$ act as unitary and $i \mathbf{W}$ and $\mathbf{X}-\mathbf{W}$ as selfadjoint operators. 
Proof Let $\mathcal{H}_{R}$ be the subspace of the inner product space $\mathcal{H}_{0}$ spanned by vectors $e_{\mu, \xi, \zeta}$ such that

$$
\mu=x+\frac{2 \pi i k}{N}, \xi=e^{\frac{2 \pi i k}{N}}, \zeta=e^{\frac{2 \pi i m}{N}}, \text { for } x \in \mathbb{R}, k, m \in \mathbb{Z} .
$$

One checks that $\mathcal{H}_{R}$ is closed under the action of $\mathcal{A}$ on $\mathcal{H}_{0}$ defined in 3.1.5 that is $\mathcal{H}_{R}$ is an $\mathcal{A}$-submodule. We also define the action by $\mathbf{W}$

$$
\mathbf{W}: e_{\mu, \xi, \zeta} \mapsto \frac{2 \pi i k}{N} e_{\mu, \xi, \zeta}
$$

for $\mu=x+\frac{2 \pi i k}{N}$. This obviously agrees with the defining relations of $\mathcal{A}^{\#}$. So $\mathcal{H}_{R}$ is an $\mathcal{A}^{\#}$-submodule of $\mathcal{H}_{0}$.

Now $\mathbf{U}$ and $\mathbf{V}$ are unitary operators on $\mathcal{H}_{R}$ since they transform the orthonormal basis into itself. $\mathbf{Y}$ is unitary since its eigenvectors form the orthonormal basis and the corresponding eigenvalues are of absolute value 1. $i \mathbf{W}$ and $\mathbf{X}-\mathbf{W}$ are selfadjoint since their eigenvalues on the orthonormal basis are the reals $-\frac{2 \pi k}{N}$ and $x$, correspondingly.

Proposition The action of the algebras $\mathcal{A}$ and $\mathcal{A}^{\#}$ on $\mathcal{H}_{R}$ are faithful, that is an operator $T$ of the algebra annihilates $\mathcal{H}_{R}$ if and only if $T=0$.

Proof We will prove the statement for $\mathcal{A}$. The proof for $\mathcal{A}^{\#}$ is similar.

Using the defining relations (7) we can represent

$$
T=\sum_{i \in I} c_{i} \mathbf{X}^{i_{1}} \mathbf{Y}^{i_{2}} \mathbf{Z}^{i_{3}} \mathbf{U}^{i_{4}} \mathbf{V}^{i_{5}} \mathbf{E}^{i_{6}}
$$

for some finite $I \subset \mathbb{Z}^{6}, i=\left\langle i_{1} \ldots i_{6}\right\rangle$ and $c_{i} \in \mathbb{C}$.

Given an element $e_{\mu, \xi, \zeta}$ of the basis, the action of $T$ on it produces

$$
T e_{\mu, \xi, \zeta}=\sum_{i \in I} c_{i} \mathbf{X}^{i_{1}} \mathbf{Y}^{i_{2}} \mathbf{Z}^{i_{3}} e_{\mu(i), \xi(i), \zeta(i)}
$$

where

$$
e_{\mu(i), \xi(i), \zeta(i)}=\mathbf{U}^{i_{4}} \mathbf{V}^{i_{5}} \mathbf{E}^{i_{6}} e_{\mu, \xi, \zeta}
$$

is a basis element by definition of the action of $\mathbf{U}$ and $\mathbf{V}$, moreover one can check that $e_{\mu(i), \xi(i), \zeta(i)}$ are distinct for distinct $\mathbf{U}^{i_{4}} \mathbf{V}^{i_{5}} \mathbf{E}^{i_{6}}$.

Since the basis elements are eigenvectors of $\mathbf{X}, \mathbf{Y}$ and $\mathbf{Z}$

$$
T e_{\mu, \xi, \zeta}=\sum_{i \in I} c_{i} \cdot d_{i}(\mu, \xi, \zeta) e_{\mu(i), \xi(i), \zeta(i)}
$$

for some nonzero $d_{i}(\mu, \xi, \zeta) \in \mathbb{C}$.

Now assume that $T$ annihilates $\mathcal{H}_{R}$. Then the right-hand-side of the above must be zero and by linear independence all $c_{i} \cdot d_{i}(\mu, \xi, \zeta)=0$, which can only happen if all $c_{i}=0$ and $T=0 . \square$

Corollary The *-operation on the generators of $\mathcal{A}^{\#}$ defined above extends uniquely to *-operation on the whole $\mathcal{A}^{\#}$ and $\left(\mathcal{A}^{\#}, *\right)$ satisfies all the identities of a $\mathbb{C}$-algebra with adjoints. Moreover, since $\mathcal{A}^{\#}$ has a faithful representation on an inner product space we can introduce the usual 
operator norm on $\mathcal{A}^{\#}$ with $\mathbf{Y}, \mathbf{Z}, \mathbf{W}, \mathbf{U}$ and $\mathbf{V}$ bounded operators and $\mathbf{X}$ unbounded.

Remark 1 Our choice of the $C^{*}$-structure on $\mathcal{A}^{\#}$ has been motivated by

(i) the need to encode the fact that the relevant $e_{\mu, \xi, \zeta}$ must be 'real oriented', that is $\mathrm{bd} \mu=\xi$;

(ii) the natural interpretation of the band function and the related function w (for $a \in i \mathbb{R}$ and $b \in \mathbb{R}$ and $N \rightarrow \infty$ ) as functions indicating when $\mu$ is 'almost real'. More precisely, as remarked in 3.1 .3 bd can be interpreted, for $a=\frac{2 \pi i}{N}, b \in \mathbb{R}$, as

$$
\operatorname{bd}(x+2 \pi i y)=\exp 2 \pi i \frac{[y N]}{N},
$$

where $x, y \in \mathbb{R}$, and $[y N]$ is the entire part of $y N$. Since $[y N] / N$ converges to $y$ the condition $\mathrm{bd} \mu=1$ says that $\mu$ is 'almost real'.

Remark 2 The natural interpretation of the band function is used in Section 4 to obtain 'the classical limit' $\mathrm{P}_{\infty}$ of the $\mathrm{P}_{N}$.

Comments 1.We have seen that in the representation $\mathcal{H}_{R}$ the $e_{\mu, \xi, \zeta}$ are eigenvectors of the self-adjoint operator $\mathbf{X}-\mathbf{W}$. So in physics jargon $\left\langle e_{\mu, \xi, \zeta}\right\rangle$ would be called states.

2. The discrete nature of the imaginary part of $\mu$ in (8) is necessitated by two conditions: the interpretation of $*$ as taking adjoints and the noncontinuous form of the band function. The first condition is crucial for any physical interpretation and the second one follows from the description of the Zariski structure $\mathrm{P}_{N}$. Comparing this to the real differentiable structure $\mathrm{P}_{\infty}$ constructed in Section 4 as the limit of the $\mathrm{P}_{N}$ we suggest to interpret the latter along with its representation via $\mathcal{A}$ in this section as the quantisation of the former.

\subsection{The algebraic torus case}

3.2.1 Let $\mathrm{F}$ be an algebraically closed field of any characteristic prime to $N$ an $a, b \in \mathrm{F}^{*}$ be multiplicatively independent.

$G$ acts on $\mathrm{F}^{*}$ :

$$
u x=a x, v x=b x .
$$

Taking $M$ to be $\mathrm{F}^{*}$ this determines, by subsection 2, a presmooth nonalgebraic Zariski curve $\tilde{M}$ which from now on we denote $\mathrm{T}_{N}$.

The correspondent definition for the covering map p : $\tilde{M}=T_{N} \rightarrow$ $M=\mathrm{F}^{*}$ then gives us

$$
\mathbf{p}(\mathbf{u} t)=a \mathbf{p}(t), \quad \mathbf{p}(\mathbf{v} t)=b \mathbf{p}(t) .
$$

We also note that there exists the well-defined function $\mathbf{p}^{\prime}: T_{N} \rightarrow \mathrm{F}$ given by

$$
\mathbf{p}^{\prime}(t) \mathbf{p}(t)=1
$$


For the rest of the section fix $\alpha=a^{\frac{1}{N}}$ and $\beta=b^{\frac{1}{N}}$, roots of $a$ and $b$ of order $N$.

\subsubsection{Semi-definable functions in $\mathrm{T}_{N}$.}

Lemma There exist functions

$$
\mathbf{x}, \mathbf{x}^{\prime}, \mathbf{y}: T_{N} \rightarrow \mathrm{F}
$$

satisfying the following functional equations, for any $t \in T_{N}$,

$$
\begin{gathered}
\mathbf{x}^{N}(t)=\mathbf{p}(t), \mathbf{x}(\mathbf{u} t)=\alpha \mathbf{x}(t), \mathbf{x}(\mathbf{v} t)=\beta \mathbf{y}(t) \mathbf{x}(t) \\
\mathbf{x}(t) \mathbf{x}^{\prime}(t)=1 \\
\mathbf{y}^{N}(t)=1, \mathbf{y}(\mathbf{u} t)=\epsilon \mathbf{y}(t), \mathbf{y}(\mathbf{v} t)=\mathbf{y}(t)
\end{gathered}
$$

Proof Choose a subset $S \subseteq \mathrm{F}^{*}$ of representatives of $G$-orbits, that is $\mathrm{F}=G \cdot S$. By construction 2.0.1 we can identify $T_{N}=\tilde{M}$ with $\tilde{G} \times S$ in such a way that $\mathbf{p}(\gamma s)=\operatorname{pr}(\gamma) \cdot s$. This means that, for any $s \in S$ and $t \in \tilde{G} \cdot s$ of the form $t=\mathbf{u}^{m} \mathbf{v}^{n}[\mathbf{u}, \mathbf{v}]^{\ell} \cdot s$,

$$
\mathbf{p}\left(\mathbf{u}^{m} \mathbf{v}^{n}[\mathbf{u}, \mathbf{v}]^{\ell} \cdot s\right):=a^{n} \cdot b^{m} \cdot s .
$$

Fix (randomly) a root $s^{\frac{1}{N}}$ of order $N$ for each $s \in S$. Set

$$
\mathbf{x}\left(\mathbf{u}^{m} \mathbf{v}^{n}[\mathbf{u}, \mathbf{v}]^{\ell} \cdot s\right):=\alpha \cdot \beta \cdot \epsilon^{-\ell} s^{\frac{1}{N}}
$$

Set also

$$
\mathbf{y}\left(\mathbf{u}^{m} \mathbf{v}^{n}[\mathbf{u}, \mathbf{v}]^{\ell} \cdot s\right):=\epsilon^{m} .
$$

This satisfies (11)- (13).

Remark. Notice, that it follows from (11) and (13) that

$\mathbf{x}([\mathbf{u}, \mathbf{v}] t)=\epsilon^{-1} \mathbf{x}(t)$

$\mathbf{y}([\mathbf{u}, \mathbf{v}] t)=\mathbf{y}(t)$.

3.2.3 Define the angular function on $\mathrm{F}$ as a function ang $: \mathrm{F} \rightarrow \mathrm{F}[N]$. Set for $\lambda \in \mathrm{F}$

$$
\operatorname{ang}(\lambda)=\mathbf{y}(t), \text { if } \mathbf{p}(t)=\lambda .
$$

This is well-defined by the remark in 3.2 .2

Acting by $\mathbf{u}$ on $t$ and using (9) and (13) we have

$$
\operatorname{ang}(a \lambda)=\epsilon \operatorname{ang} \lambda \text {. }
$$

Acting by $\mathbf{v}$ we obtain

$$
\operatorname{ang}(b \lambda)=\operatorname{ang} \lambda .
$$

Proposition The structure $\mathrm{T}_{N}$ is definable in

$$
(\mathrm{F},+, \cdot, \text { ang }) \text {. }
$$

Indeed, set $T_{N}=\mathrm{F}^{*}$ and define the maps

$$
\mathbf{p}(t):=t^{N}
$$


and

$$
\mathbf{u}(t):=\alpha t, \mathbf{v}(t):=\operatorname{ang}\left(t^{N}\right) \beta t .
$$

One checks easily that

$$
\mathbf{v u}(t)=\epsilon \cdot \mathbf{u v}(t)
$$

and so the action of $\tilde{G}$ is well-defined and that (9) holds.

Remark 1 Assuming that $\mathrm{F}=\mathbb{C}$ and $\epsilon=\exp \left(\frac{2 \pi i}{N}\right)$, let for an $r \in \mathbb{R}$,

$$
a=\exp \left(\frac{2 \pi i}{N}+r\right), \quad \text { and } b \in \mathbb{R}_{+}, b \neq 1 .
$$

Then we may define, for $z \in \mathbb{C}$,

$$
\text { ang } z:=\exp \left(\frac{2 \pi i}{N}\left[\frac{N}{2 \pi} \arg z\right]\right)
$$

This is a well-defined function satisfying also (14) and (15), and so $\mathrm{T}_{N}$ over $\mathbb{C}$ is definable in $\mathbb{C}$ equipped with the measurable but not continuous function above.

It is also interesting to remark that, for this angular function,

$$
\left|\arg z-\frac{2 \pi}{N}\left[\frac{N}{2 \pi} \arg z\right]\right| \leq \frac{2 \pi}{N}
$$

and so ang $z$ converges uniformly on $z$ to $\exp (i \arg z)$ as $N$ tends to $\infty$.

Remark 2 In the context of noncommutative geometry it is interesting to see whether there exists an abstract, model-theoretic interpretation of ang which allows a measure theory for the semi-definable functions introduced above. David Evans proved the following theorem.

Theorem (D.Evans [E]) The class of fields $(\mathrm{F},+, \cdot$, ang) of a fixed characteristic endowed with a function ang satisfying (14) and (15) has a model companion, which is a supersimple theory. The models of the theory allow a nontrivial finite measure such that all definable sets are measurable.

3.2.4 The space of semi-definable functions and the operator algebra.

Let $\mathcal{H}$ be an F-algebra containing all the functions $T_{N} \rightarrow \mathrm{F}$ which are definable in $\mathrm{T}_{N}$ expanded by $\mathbf{x}$ and $\mathbf{y}$.

We define linear operators $\mathbf{X}, \mathbf{X}^{-1}, \mathbf{Y}, \mathbf{U}$ and $\mathbf{V}$ on $\mathcal{H}$ :

$$
\begin{aligned}
& \mathbf{X}: \psi(t) \mapsto \mathbf{x}(t) \cdot \psi(t) \\
& \mathbf{X}^{-1}: \psi(t) \mapsto \mathbf{x}^{\prime}(t) \cdot \psi(t), \\
& \mathbf{Y}: \psi(t) \mapsto \mathbf{y}(t) \cdot \psi(t), \\
& \mathbf{U}: \psi(t) \mapsto \psi(\mathbf{u} t) \\
& \mathbf{V}: \psi(t) \mapsto \psi(\mathbf{v} t)
\end{aligned}
$$

Denote $\tilde{G}^{*}$ the group generated by the operators $\mathbf{U}, \mathbf{V}, \mathbf{U}^{-1}, \mathbf{V}^{-1}$, denote $\mathfrak{X}_{\epsilon}$ the $\mathrm{F}$-algebra $\mathrm{F}\left[\mathbf{X}, \mathbf{X}^{-1}, \mathbf{Y}\right]$ and $\mathcal{A}_{\epsilon}$ (or simply $\mathcal{A}$ ) the extension of the F algebra $\mathfrak{X}_{\epsilon}$ by $\tilde{G}^{*}$. 
The generators of the algebra $\mathcal{A}_{\epsilon}$ obviously satisfy the following relations, for $\mathbf{E}$ standing for the commutator $[\mathbf{U}, \mathbf{V}]$,

$$
\begin{aligned}
& \mathbf{X Y}=\mathbf{Y X} ; \\
& \mathbf{Y}^{N}=1 ; \mathbf{X X} \mathbf{X}^{-1}=1 ; \\
& \mathbf{X U}=\alpha^{-1} \mathbf{U X} ; \\
& \mathbf{X V}=\beta^{-1} \mathbf{Y}^{-1} \mathbf{V X} ; \\
& \mathbf{Y U}=\epsilon^{-1} \mathbf{U Y} ; \\
& \mathbf{Y V}=\mathbf{V Y} ; \\
& \mathbf{U E}=\mathbf{E} \mathbf{U} ; \mathbf{V}=\mathbf{E V} ; \mathbf{E}^{N}=1 .
\end{aligned}
$$

We prove later on, in the Proposition and Corollary of 3.1.6 that the algebra determined by the relations (17) is exactly $\mathcal{A}$ and so the definition of $\mathcal{A}$ does not depend on the arbitrariness in the construction of $\mathcal{H}$.

3.2.5 Let $\operatorname{Max}(\mathfrak{X})$ be the set of isomorphism classes of 1-dimensional irreducible $\mathfrak{X}$-modules.

Lemma $1 \operatorname{Max}(\mathfrak{X})$ can be represented by 1 -dimensional modules $\left\langle e_{\mu, \xi}\right\rangle$ $\left(=\mathrm{F} e_{\mu, \xi}\right)$ for $\mu \in \mathrm{F}, \xi \in \mathrm{F}[N]$, defined by the action on the corresponding generating vector:

$$
\mathbf{X} e_{\mu, \xi}=\mu e_{\mu, \xi}, \quad \mathbf{Y} e_{\mu, \xi}=\xi e_{\mu, \xi}
$$

Proof This is a standard fact of commutative algebra. $\square$

Now we introduce an infinite-dimensional $\mathcal{A}$-module $\mathcal{H}_{0}$. As a vector space $\mathcal{H}_{0}$ is spanned by $\left\{e_{\mu, \xi}: \mu \in \mathrm{F}, \xi \in \mathrm{F}[N]\right\}$. The action of the generators of $\mathcal{A}$ on $\mathcal{H}_{0}$ is defined on $e_{\mu, \xi}$ in accordance with the defining relations of $\mathcal{A}$. So, since

$$
\begin{gathered}
\mathbf{X U} e_{\mu, \xi}=\alpha^{-1} \mathbf{U X} e_{\mu, \xi}=\alpha^{-1} \mu \mathbf{U} e_{\mu, \xi}, \\
\mathbf{Y} \mathbf{U} e_{\mu, \xi}=\epsilon^{-1} \mathbf{U Y} e_{\mu, \xi}=\epsilon^{-1} \xi \mathbf{U} e_{\mu, \xi},
\end{gathered}
$$

and

$$
\begin{gathered}
\mathbf{X V} e_{\mu, \xi}=\beta^{-1} \mathbf{V} \mathbf{Y}^{-1} \mathbf{X} e_{\mu, \xi}=\beta^{-1} \xi^{-1} \mu \mathbf{V} e_{\mu, \xi} \\
\mathbf{Y V} e_{\mu, \xi}=\mathbf{V Y} e_{\mu, \xi}=\xi \mathbf{V} e_{\mu, \xi}
\end{gathered}
$$

we set

$$
\mathbf{U} e_{\mu, \xi}:=e_{\nu, \zeta}, \text { with } \nu=\alpha \mu, \quad \zeta=\epsilon^{-1} \xi
$$

and

$$
\mathbf{V} e_{\mu, \xi}:=e_{\nu, \zeta}, \text { with } \nu=\beta \xi^{-1} \mu, \quad \zeta=\xi .
$$

We may now identify $\operatorname{Max}(\mathfrak{X})$ as the family of 1 -dimensional $\mathfrak{X}$-eigenspaces of $\mathcal{H}_{0}$.

Assuming $\mathrm{F}$ is endowed with an angular function ang we call $\langle\mu, \xi\rangle$ as above positively oriented if

$$
\operatorname{ang} \mu^{N}=\xi \text {. }
$$


Correspondingly, we call the $\mathfrak{X}$-module (state) $\left\langle e_{\mu, \xi}\right\rangle$ positively oriented if $\langle\mu, \xi\rangle$ is. $\mathcal{H}_{0}^{+}$will denote the linear subspace of $\mathcal{H}_{0}$ spanned by the positively oriented states $\left\langle e_{\mu, \xi}\right\rangle$. We denote $\operatorname{Max}^{+}(\mathfrak{X})$ the family of 1dimensional positively oriented $\mathfrak{X}$-eigenspaces of $\mathcal{H}_{0}$, or states as such things are referred to in physics literature.

Lemma 2 (i) $\langle\mu, \xi\rangle$ is positively oriented if and only if

$$
\langle\mu, \xi\rangle=\langle\mathbf{x}(t), \mathbf{y}(t)\rangle
$$

for some $t \in T$.

(ii) $\mathcal{H}_{0}^{+}$is invariant under the action of $\mathbf{U}$ and $\mathbf{V}$, so is an $\mathcal{A}$-module.

Proof (i) Indeed, since $p$ is a surjection, there is $t^{\prime} \in T$ such that $\mathbf{p}\left(t^{\prime}\right)=$ $\mu^{N}$. Hence, by the definition of $\mathbf{x}\left(t^{\prime}\right)$ and $\operatorname{ang}\left(t^{\prime}\right)$ we have $\mathbf{x}\left(t^{\prime}\right)=\epsilon^{k} \mu$, $\mathbf{y}\left(t^{\prime}\right)=\xi$, for some $k$. By the Remark in 3.2 .2 we have $\mathbf{x}\left([\mathbf{u}, \mathbf{v}]^{k} t^{\prime}\right)=$ $\epsilon^{-k} \mathbf{x}\left(t^{\prime}\right)=\mu$ and $\mathbf{y}\left([\mathbf{u}, \mathbf{v}]^{k} t^{\prime}\right)=\mathbf{y}\left(t^{\prime}\right)=\xi$. So $t=[\mathbf{u}, \mathbf{v}]^{k} t^{\prime}$ is as required.

(ii) Immediate by the definition of the action. $\square$

Remark 2 It is immediate from the Lemma and Remark 1 that all the positively oriented $e_{\mu, \xi}$ are represented by the Dirac functions $\delta_{t}, t \in T_{N}$.

Theorem 3 (i) There is a bijective correspondence $\Xi: \operatorname{Max}^{+}(\mathfrak{X}) \rightarrow T_{N}$ between the set of positively oriented states and $T_{N}$.

(ii) The action of $\tilde{G}^{*}$ on $\mathcal{H}_{0}$ induces an action on $\operatorname{Max}(\mathfrak{X})$ and leaves $\operatorname{Max}^{+}(\mathfrak{X})$ setwise invariant. The correspondence $\Xi$ transfers anti-isomorphically the natural action of $\tilde{G}^{*}$ on $\operatorname{Max}^{+}(\mathfrak{X})$ to the natural action of $\tilde{G}$ on $T_{N}$.

(iii) The map

$$
\mathbf{p}_{\mathfrak{X}}:\left\langle e_{\mu, \xi}\right\rangle \mapsto \mu^{N}
$$

is a $N$-to-1-surjection $\mathrm{Max}^{+}(\mathfrak{X}) \rightarrow \mathrm{F}$ such that

$$
\left(\operatorname{Max}^{+}(\mathfrak{X}), \mathbf{U}, \mathbf{V}, \mathbf{p}_{\mathfrak{X}}, \mathrm{F}\right) \cong \Xi\left(T_{N}, \mathbf{u}, \mathbf{v}, \mathbf{p}, \mathrm{F}\right) .
$$

Proof (i) Immediate by Lemma 2 of 3.2 .5

(ii) Indeed, by the definition above the action of $\mathbf{U}$ and $\mathbf{V}$ corresponds to the action on positively oriented pairs:

$$
\begin{aligned}
& \mathbf{u}: e_{\mathbf{x}(t), \mathbf{y}(t)} \mapsto \mathbf{U}^{-1} e_{\mathbf{x}(t), \mathbf{y}(t)}=e_{\alpha \mathbf{x}(t), \epsilon \mathbf{y}(t)}=e_{\mathbf{x}(\mathbf{u} t), \mathbf{y}(\mathbf{u} t)}, \\
& \mathbf{v}: e_{\mathbf{x}(t), \mathbf{y}(t)} \mapsto \mathbf{V}^{-1} e_{\mathbf{x}(t), \mathbf{y}(t)}=e_{\beta \mathbf{y}(t) \mathbf{x}(t), \mathbf{y}(t)}=e_{\mathbf{x}(\mathbf{v} t), \mathbf{y}(\mathbf{v} t)} .
\end{aligned}
$$

(iii) Immediate from (i) and (ii)

\subsection{6 $C^{*}$-structure}

We add to 3.2 .2 the new semi-definable function $\mathbf{w}$ satisfying, for some $\delta$, such that $\delta^{N}=\epsilon$,

$$
\mathbf{y}=\mathbf{w}^{N}, \quad \mathbf{w}(\mathbf{u} t)=\delta \mathbf{w}(t), \quad \mathbf{w}(\mathbf{v} t)=\mathbf{y}(t)^{-1} \mathbf{w}(t) .
$$

In accordance with 3.2 .2 we can define

$$
\mathbf{w}\left(\mathbf{u}^{m} \mathbf{v}^{n}[\mathbf{u}, \mathbf{v}]^{l}\right)=\delta^{m} \epsilon^{l} .
$$


Now we introduce

$$
\text { ang } x:=\mathbf{w}(t) \text {, for } x=\mathbf{x}(t) \text {. }
$$

Since $\mathbf{x}$ is a bijection this is well-defined on F. Moreover, using the unique representation

$$
x=\mathbf{x}\left(\mathbf{u}^{m} \mathbf{v}^{n}[\mathbf{u}, \mathbf{v}]^{l}\right)=\alpha^{m} \beta^{n} \epsilon^{-l} s^{\frac{1}{N}}
$$

of 3.2 .2 we have

$$
\operatorname{ang}\left(\alpha^{m} \beta^{n} \epsilon^{-l} s^{\frac{1}{N}}\right)=\delta^{m} \epsilon^{l} \text {. }
$$

Taking $a=\epsilon \rho, \rho \in \mathbb{R}_{+}$(positive reals), $\rho \neq 1$, as suggested in 3.2.3. and $\alpha^{-1} \delta \in \mathbb{R}_{+}$, we have

$$
\operatorname{ang}(\alpha x)=\delta \operatorname{ang} x, \quad \operatorname{ang}(\beta x)=\operatorname{ang} x .
$$

Extend the list of operators on $\mathcal{H}$ to include

$$
\mathbf{W}: \psi \mapsto \mathbf{W} \cdot \psi
$$

Obviously $\mathbf{W}$ commutes with $\mathbf{X}$. As in 3.2 .5 denote $e_{\mu, w}$ an eigenvector of $\mathbf{X}$ and $\mathbf{W}$ with eigenvalues $\mu$ and $w$ correspondingly. The action of $\mathbf{U}$ and $\mathbf{V}$ is defined on $e_{\mu, w}$ similarly to 3.2 .5

$$
\begin{aligned}
\mathbf{U}: e_{\mu, w} & \mapsto e_{\alpha^{-1} \mu, \delta^{-1} w} \\
\mathbf{V}: e_{\mu, w} & \mapsto e_{\beta^{-1} w^{-N} \mu, w^{1-N}}
\end{aligned}
$$

Consider the algebra $\mathcal{A}$ as a $C^{*}$-algebra with the condition that $\mathbf{X} \mathbf{W}^{-1}$ is selfadjoint and $\mathbf{W}, \mathbf{U}$ and $\mathbf{V}$ are unitary.

Set

$$
\mathbf{W}^{*}:=\mathbf{W}^{-1}, \mathbf{U}^{*}:=\mathbf{U}^{-1}, \mathbf{V}^{*}:=\mathbf{V}^{-1}
$$

that is define these operators as unitary. Set

$$
\mathbf{X}^{*}:=\mathbf{W}^{-1} \mathbf{X} \mathbf{W}^{-1}=\mathbf{X} \mathbf{W}^{-2},
$$

so

$$
\left(\mathbf{X} \mathbf{W}^{-1}\right)^{*}=\mathbf{W}^{*-1} \mathbf{X}^{*}=\mathbf{W} \mathbf{X}^{*}=\mathbf{X} \mathbf{W}^{-1}
$$

that is $\mathbf{X} \mathbf{W}^{-1}$ is selfadjoint.

Of course

$$
[\mathbf{U}, \mathbf{V}]^{*}=[\mathbf{U}, \mathbf{V}]^{-1}
$$

so $[\mathbf{U}, \mathbf{V}]$ is unitary as well.

Lemma There is an inner product space $\mathcal{H}_{+}$with the faithful action of $\mathcal{A}$ on it such that $*$ corresponds to taking adjoint operators.

Proof Consider $\mathcal{H}_{+} \subseteq \mathcal{H}$ generated by all $e_{\mu, w}$ satisfying the condition

$$
\mu \cdot w^{-1} \in \mathbb{R}_{+}, w=\exp \frac{2 \pi i k}{N^{2}}, \text { for } k \in \mathbb{Z} .
$$

We introduce the inner product in $\mathcal{H}_{+}$assuming the $e_{\mu, w}$ to form an orthonormal basis. 
Now, by definition $\mathbf{X} \mathbf{W}^{-1}$ acts as a positive selfadjoint operator

$$
\mathbf{X} \mathbf{W}^{-1}: e_{\mu, w} \mapsto \mu w^{-1} e_{\mu, w} .
$$

$\mathbf{W}$ acts as unitary since $w$ is a root of unity. $\mathbb{R}_{+}$.

$\mathcal{H}_{+}$is closed under $\mathbf{U}$ and $\mathbf{V}$ since $\alpha^{-1} \mu \delta w^{-1}$ and $\beta^{-1} \mu \delta w^{-1}$ are in

The fact that the action is faithful (that is the only operator that annihilates $\mathcal{H}$ is 0 ) is essentially proved in the Proposition and Corollary of 3.1 .6

Comment Using the representation on $\mathcal{H}_{+}$one clearly can interpret the angular function ang $\mu$ as $\exp \arg \mu$, for $\mu$ satisfying (19). For general $\mu$ we can use the interpretation as in 3.2 .3

$$
\text { ầg } \mu=\exp \frac{2 \pi i}{N^{2}}\left[\frac{N^{2}}{2 \pi} \arg \mu\right] \text {, }
$$

where $[r]$ stands for the integer part of a real number $r$. Of course, we stress again that ang $\mu$ is very well approximated by $\exp \arg \mu$ since

$$
\left|\frac{2 \pi i}{N^{2}}\left[\frac{N^{2}}{2 \pi} \arg \mu\right]-\arg \mu\right| \leq \frac{2 \pi}{N^{2}} .
$$

In other words, the condition on the states being positively oriented in Theorem 3 is similar to conditions usually stated in terms of $C^{*}$-algebras. This must justify the name $*$-functions for ang, ang and bd.

\section{The metric limit}

Our aim in this section is to find an interpretation of the limit, as $N$ tends to $\infty$, of structures $\mathrm{T}_{N}$ or $\mathrm{P}_{N}$ in "classical" terms. "Classical" here is supposed to mean " using function and relations given in terms of real manifolds and analytic functions". Of course, we have to define the meaning of the "limit" first. We found a satisfactory solution to this problem in case of $\mathrm{P}_{N}$ which is presented below.

4.0.7 First we want to establish a connection of the group $\tilde{G}_{N}$ with the integer Heisenberg group $H(\mathbb{Z})$ which is the group of matrices of the form

$$
\left(\begin{array}{lll}
1 & k & m \\
0 & 1 & l \\
0 & 0 & 1
\end{array}\right)
$$

with $k, l, m \in \mathbb{Z}$. More precisely, $\tilde{G}_{N}$ is isomorphic to the group

$$
H(\mathbb{Z})_{N}=H(\mathbb{Z}) / N . Z,
$$

where $N . Z$ is the central subgroup

$$
N . Z=\left\{\left(\begin{array}{ccc}
1 & 0 & N m \\
0 & 1 & 0 \\
0 & 0 & 1
\end{array}\right): m \in \mathbb{Z}\right\}
$$


Similarly the real Heisenberg group $H(\mathbb{R})$ is defined as the group of matrices of the form (20) with $k, l, m \in \mathbb{R}$. The analogue (or the limit case) of $H(\mathbb{Z})_{N}$ is the factor-group

$$
H(\mathbb{R})_{\infty}:=H(\mathbb{R}) /\left(\begin{array}{ccc}
1 & 0 & \mathbb{Z} \\
0 & 1 & 0 \\
0 & 0 & 1
\end{array}\right)
$$

In fact there is the natural group embedding

$$
i_{N}:\left(\begin{array}{lll}
1 & k & m \\
0 & 1 & \ell \\
0 & 0 & 1
\end{array}\right) \mapsto\left(\begin{array}{lll}
1 & \frac{k}{\sqrt{N}} & \frac{m}{N} \\
0 & 1 & \frac{\ell}{\sqrt{N}} \\
0 & 0 & 1
\end{array}\right)
$$

inducing the embedding $H(\mathbb{Z})_{N} \subset H(\mathbb{R})_{\infty}$.

Notice the following

Lemma 1 Given the embedding $i_{N}$ for every $\langle u, v, w\rangle \in H(\mathbb{R})_{\infty}$ there is $\left\langle\frac{k}{\sqrt{N}}, \frac{\ell}{\sqrt{N}}, \frac{m}{N}\right\rangle \in i_{N}\left(H(\mathbb{Z})_{N}\right)$ such that

$$
\left|u-\frac{k}{\sqrt{N}}\right|+\left|v-\frac{\ell}{\sqrt{N}}\right|+\left|w-\frac{m}{N}\right|<\frac{3}{\sqrt{N}} .
$$

In other words, the distance (given by the sum of absolute values) between any point of $H(\mathbb{R})_{\infty}$ and the set $i_{N}\left(H(\mathbb{Z})_{N}\right)$ is at most $3 / \sqrt{N}$. Obviously, also the distance between any point of $i_{N}\left(H(\mathbb{Z})_{N}\right)$ and the set $H(\mathbb{R})_{\infty}$ is 0 , because of the embedding. In other words, this defines that the Hausdorff distance between the two sets is at most $3 / \sqrt{N}$.

In situations when the pointwise distance between sets $M_{1}$ and $M_{2}$ is defined we also say that the Hausdorff distance between two $L$-structures on $M_{1}$ and $M_{2}$ is at most $\alpha$ if the Hausdorff distance between the universes $M_{1}$ and $M_{2}$ as well as between $R\left(M_{1}\right)$ and $R\left(M_{2}\right)$, for any $L$-predicate or graph of an $L$-operation $R$, is at most $\alpha$.

Finally, we say that an $L$-structure $M$ is the Hausdorff limit of $L$ structures $M_{N}, N \in \mathbb{N}$, if for each positive $\alpha$ there is $N_{0}$ such that for all $N>N_{0}$ the distance between $M_{N}$ and $M$ is at most $\alpha$.

Remark It makes sense to consider the similar notion of Gromov-Hausdorff distance and Gromov-Hausdorff limit.

Lemma 2 The group structure $H(\mathbb{R})_{\infty}$ is the Hausdorff limit of its substructures $H(\mathbb{Z})_{N}$, where the distance is defined by the embeddings $i_{N}$.

Proof Lemma 1 proves that the universe of $H(\mathbb{R})_{\infty}$ is the limit of the corresponding sequence. Since the group operation is continuous in the topology determined by the distance, the graphs of the group operations converge as well. $\square$ 
4.0.8 Given nonzero real numbers $a, b, c$ the integer Heisenberg group $H(\mathbb{Z})$ acts on $\mathbb{R}^{3}$ as follows:

$$
\langle k, l, m\rangle\langle x, y, s\rangle=\langle x+a k, y+b l, s+a c k y+a b c m\rangle
$$

where $\langle k, l, m\rangle$ is the matrix (20).

We can also define the action of $H(\mathbb{Z})$ on $\mathbb{C} \times S^{1}$, equivalently on $\mathbb{R} \times \mathbb{R} \times \mathbb{R} / \mathbb{Z}$, as follows

$$
\langle k, l, m\rangle\langle x, y, \exp 2 \pi i s\rangle=\langle x+a k, y+b l, \exp 2 \pi i(s+a c k y+a b c m)\rangle
$$

where $x, y, s \in \mathbb{R}$.

In the discrete version intended to model 3.1 .1 we consider $\frac{q}{N}, q \in \mathbb{Z}$, in place of $s \in \mathbb{R}$ and take $a=b=\frac{1}{\sqrt{N}}$. We replace (22) by

$$
\langle k, l, m\rangle\left\langle x, y, e^{\frac{2 \pi i q}{N}}\right\rangle=\left\langle x+\frac{k}{\sqrt{N}}, y+\frac{\ell}{\sqrt{N}}, \exp 2 \pi i \frac{q+k[y \sqrt{N}]+m}{N}\right\rangle
$$

Check that this is still an action:

$$
\begin{gathered}
\left\langle k^{\prime}, \ell^{\prime}, m^{\prime}\right\rangle\left(\langle k, \ell, m\rangle\left\langle x, y, \exp \frac{2 \pi i q}{N}\right\rangle\right)= \\
\left\langle k^{\prime}, \ell^{\prime}, m^{\prime}\right\rangle\left\langle x+\frac{k}{\sqrt{N}}, y+\frac{\ell}{\sqrt{N}}, \exp 2 \pi i \frac{q+k[y \sqrt{N}]+m}{N}\right\rangle= \\
\left\langle x+\frac{k}{\sqrt{N}}+\frac{k^{\prime}}{\sqrt{N}}, y+\frac{\ell}{\sqrt{N}}+\frac{\ell^{\prime}}{\sqrt{N}}, \exp 2 \pi i \frac{q+k[y \sqrt{N}]+m+k^{\prime}\left[\left(y+\frac{\ell}{\sqrt{N}}\right) \sqrt{N}\right]+m^{\prime}}{N}\right\rangle= \\
\left\langle x+\frac{k+k^{\prime}}{\sqrt{N}}, y+\frac{\ell+\ell^{\prime}}{\sqrt{N}}, \exp 2 \pi i \frac{q+\left(k+k^{\prime}\right)[y \sqrt{N}]+k^{\prime} l+m+m^{\prime}}{N}\right\rangle= \\
\left(\left\langle k^{\prime}, l^{\prime}, m^{\prime}\right\rangle\langle k, l, m\rangle\right)\left\langle x, y, \exp \frac{2 \pi i q}{N}\right\rangle
\end{gathered}
$$

Moreover, we may take $m$ modulo $N$ in (23), that is $\langle k, l, m\rangle \in H(\mathbb{Z})_{N}$, and simple calculations similar to the above show the following

Lemma 1 The formula (23) defines the free action of $H(\mathbb{Z})_{N}$ on $\mathbb{R} \times \mathbb{R} \times \exp \frac{2 \pi i}{N} \mathbb{Z}$ (equivalently on $\mathbb{C} \times \exp \frac{2 \pi i}{N} \mathbb{Z}$ ).

We think of $\left\langle x, y, \exp \frac{2 \pi i q}{N}\right\rangle$ as an element $t$ of $P_{N}$ (see 3.1.1),$x+i y$ as $p(t) \in \mathbb{C}$. The actions $x+i y \mapsto a+x+i y$ and $x+i y \mapsto x+i(y+b)$ are obvious rational automorphisms of the affine line $\mathbb{C}$.

We interpret the action of $\langle 1,0,0\rangle$ and $\langle 0,1,0\rangle$ by (23) on $\mathbb{C} \times \exp \frac{2 \pi i}{N} \mathbb{Z}$ as $\mathbf{u}$ and $\mathbf{v}$ correspondingly. Then the commutator $[\mathbf{u}, \mathbf{v}]$ corresponds to $\langle 0,0,-1\rangle$, which is the generating element of the centre of $H(\mathbb{Z})_{N}$. In other words, the subgroup $\operatorname{gp}(\mathbf{u}, \mathbf{v})$ of $H(\mathbb{Z})_{N}$ generated by the two elements is isomorphic to $\tilde{G}$. We thus get, using Lemma 1 of 2.0 .1

Lemma 2 Under the above assumption and notation the structure on $\mathbb{C} \times \exp \frac{2 \pi i}{N} \mathbb{Z}$ in the language of 3.1.1 described by (23) is isomorphic to the example $\mathrm{P}_{N}$ of 3.1 .1 with $\mathrm{F}=\mathbb{C}$. 
Below we identify $\mathrm{P}_{N}$ with the structure above based on $\mathbb{C} \times\left\{\exp \frac{2 \pi i}{N} \mathbb{Z}\right\}$.

Note that every group word in $\mathbf{u}$ and $\mathbf{v}$ gives rise to a definable map in $\mathrm{P}_{N}$. We want introduce a uniform notation for such definable functions.

Let $\alpha$ be a monotone nondecreasing converging sequence of the form

$$
\alpha=\left\{\frac{k_{N}}{\sqrt{N}}: k_{N}, N \in \mathbb{Z}, N>0\right\}
$$

We call such a sequence admissible if there is an $r \in \mathbb{R}$ such that

$$
\left|r-\frac{k_{N}}{\sqrt{N}}\right| \leq \frac{1}{\sqrt{N}} .
$$

Given $r \in \mathbb{R}$ and $N \in \mathbb{N}$ one can easily find $k_{N}$ satisfying (24) and so construct an $\alpha$ converging to $r$, which we denote $\hat{\alpha}$,

$$
\hat{\alpha}:=\lim \alpha=\lim _{N} \frac{k_{N}}{\sqrt{N}} .
$$

We denote $I$ the set of all admissible sequences converging to a real on $[0,1]$, so

$$
\{\hat{\alpha}: \alpha \in I\}=\mathbb{R} \cap[0,1] .
$$

For each $\alpha \in I$ we introduce two operation symbols $\mathbf{u}_{\alpha}$ and $\mathbf{v}_{\alpha}$. We denote $\mathrm{P}_{N}^{\#}$ the definable expansion of $\mathrm{P}_{N}$ by all such symbols with the interpretation

$$
\mathbf{u}_{\alpha}=\mathbf{u}^{k_{N}}, \quad \mathbf{v}_{\alpha}=\mathbf{v}^{k_{N}} \quad\left(k_{N} \text {-multiple of the operation }\right),
$$

if $\frac{k_{N}}{\sqrt{N}}$ stands in the $N$ th position in the sequence $\alpha$.

Note that the sequence

$$
d t:=\left\{\frac{1}{\sqrt{N}}: N \in \mathbb{N}\right\}
$$

is in $I$ and $\mathbf{u}_{d t}=\mathbf{u}, \mathbf{v}_{d t}=\mathbf{v}$ in all $\mathrm{P}_{N}^{\#}$.

4.0.9 We now define the structure $\mathrm{P}_{\infty}$ to be the structure on sorts $\mathbb{C} \times S^{1}$ (denoted $P_{\infty}$ ) and sort $\mathbb{C}$, with the field structure on $\mathbb{C}$ and the projection map $\mathbf{p}:\left\langle x, y, e^{2 \pi i s}\right\rangle \mapsto\langle x, y\rangle \in \mathbb{C}$, and definable maps $\mathbf{u}_{\alpha}$ and $\mathbf{v}_{\beta}, \alpha, \beta \in I$, acting on $\mathbb{C} \times S^{1}$ (in accordance with the action by $H(\mathbb{R})_{\infty}$ ) as follows

$$
\begin{aligned}
& \mathbf{u}_{\alpha}\left(\left\langle x, y, e^{2 \pi i s}\right\rangle\right)=\langle\hat{\alpha}, 0,0\rangle\left\langle x, y, e^{2 \pi i s}\right\rangle=\left\langle x+\hat{\alpha}, y, e^{2 \pi i(s+\hat{\alpha} y)}\right\rangle \\
& \mathbf{v}_{\beta}\left(\left\langle x, y, e^{2 \pi i s}\right\rangle\right)=\langle 0, \hat{\beta}, 0\rangle\left\langle x, y, e^{2 \pi i s}\right\rangle=\left\langle x, y+\hat{\beta}, e^{2 \pi i s}\right\rangle
\end{aligned}
$$

Theorem $1 \mathrm{P}_{\infty}$ is the Hausdorff limit of structures $\mathrm{P}_{N}^{\#}$.

Proof The sort $\mathbb{C}$ is the same in all structures.

The sort $P_{\infty}$ is the limit of its substructures $P_{N}$ since $S^{1}(=\exp i \mathbb{R})$ is the limit of $\exp \frac{2 \pi i}{N} \mathbb{Z}$ in the standard metric of $\mathbb{C}$. Also, the graph of the projection map $\mathbf{p}: P_{\infty} \rightarrow \mathbb{C}$ is the limit of $\mathbf{p}: P_{N} \rightarrow \mathbb{C}$ for the same reason.

Finally it remains to check that the graphs of $\mathbf{u}$ and $\mathbf{v}$ in $\mathrm{P}_{\infty}$ are the limits of those in $\mathrm{P}_{N}$. It is enough to see that for any $\left\langle x, y, \exp \frac{2 \pi i q}{N}\right\rangle \in P_{N}$ the result of the action by $\mathbf{u}_{\alpha}$ and $\mathbf{v}_{\beta}$ calculated in $\mathrm{P}_{N}^{\#}$ is at most at the 
distance $2 / \sqrt{N}$ from the ones calculated in $\mathrm{P}_{\infty}$, for any $\left\langle x, y, \exp \frac{2 \pi i q}{N}\right\rangle \in$ $P_{\infty}$. And indeed, the action in $\mathrm{P}_{N}^{\#}$ by definition is

$$
\begin{aligned}
& \mathbf{u}_{\alpha}:\left\langle x, y, \exp \frac{2 \pi i q}{N}\right\rangle \mapsto\left\langle x+\frac{k_{N}}{\sqrt{N}}, y, \exp \frac{2 \pi i}{N}\left(q+k_{N}[y \sqrt{N}]\right)\right\rangle \\
& \mathbf{v}_{\beta}:\left\langle x, y, \exp \frac{2 \pi i q}{N}\right\rangle \mapsto\left\langle x, y+\frac{l_{N}}{\sqrt{N}}, \exp 2 \pi i \frac{q}{N}\right\rangle
\end{aligned}
$$

Obviously,

$$
\left|\frac{k_{N} y}{\sqrt{N}}-\frac{k_{N}[y \sqrt{N}]}{N}\right|=\frac{k_{N}}{\sqrt{N}}\left|\frac{y \sqrt{N}-[y \sqrt{N}]}{\sqrt{N}}\right|<\frac{k_{N}}{\sqrt{N}} \frac{1}{\sqrt{N}} \leq \frac{1}{\sqrt{N}},
$$

which together with (24) proves that the right hand side of (26) is at the distance at most $\frac{2}{\sqrt{N}}$ from the right hand side of (25) uniformly on the point $\left\langle x, y, \exp \frac{2 \pi i q}{N}\right\rangle$.

4.0.10 The structure $P_{\infty}$ can be seen as the principal bundle over $\mathbb{R} \times \mathbb{R}$ with the structure group $U(1)$ (the rotations of $S^{1}$ ) and the projection map p. The action by the Heisenberg group allows to define a connection on the bundle. A connection determines "a smooth transition from a point in a fibre to a point in a nearby fibre". As noted above $\mathbf{u}$ and $\mathbf{v}$ in the limit process correspond to infinitesimal actions (in a nonstandard model of $\mathrm{P}_{\infty}$ ) which can be written in the form

$$
\begin{aligned}
& \mathbf{u}\left(\left\langle x, y, e^{2 \pi i s}\right\rangle\right)=\left\langle x+d x, y, e^{2 \pi i(s+y d x)}\right\rangle \\
& \mathbf{v}\left(\left\langle x, y, e^{2 \pi i s}\right\rangle\right)=\left\langle x, y+d y, e^{2 \pi i s}\right\rangle
\end{aligned}
$$

where $d x$ and $d y$ are infinitesimals equal to the $d t$ of 4.0 .8

These formulas allow to calculate the derivative of a section

$$
\psi:\langle x, y\rangle \mapsto\left\langle x, y, e^{2 \pi i s(x, y)}\right\rangle
$$

of the bundle in any direction on $\mathbb{R} \times \mathbb{R}$. In general moving infinitesimally from the point $\langle x, y\rangle$ along $x$ we get $\langle x+d x, y, \exp 2 \pi i(s+d s)\rangle$. We need to compare this to the parallel transport along $x$ given by the formulas above, $\langle x+d x, y, \exp 2 \pi i(s+y d x)\rangle$. So the difference is

$$
\langle 0,0, \exp 2 \pi i(s+d s)-\exp 2 \pi i(s+y d x)\rangle .
$$

Using the usual laws of differentiation one gets for the third term

$$
\begin{aligned}
& \exp 2 \pi i(s+d s)-\exp 2 \pi i(s+y d x)= \\
& (\exp 2 \pi i(s+d s)-\exp 2 \pi i s)-(\exp 2 \pi i(s+y d x)-\exp 2 \pi i s)= \\
& d \exp 2 \pi i s-2 \pi i y \exp 2 \pi i s d x=\left(\frac{d \exp 2 \pi i s}{d x}-2 \pi i y \exp 2 \pi i s\right) d x
\end{aligned}
$$

which gives for a section $\psi=\exp 2 \pi i s$ the following covariant derivative along $x$,

$$
\nabla_{x} \psi=\frac{d}{d x} \psi-2 \pi i y \psi=\left(\frac{d}{d x}+A_{x}\right) \psi .
$$

Similarly, the covariant derivative along $y$

$$
\nabla_{y} \psi=\frac{d}{d y} \psi=\left(\frac{d}{d y}+A_{y}\right) \psi
$$


with the second term $A_{y}=0$

The curvature of the connection is by definition the commutator

$$
\left[\nabla_{x}, \nabla_{y}\right]=\frac{d A_{y}}{d x}-\frac{d A_{x}}{d y}=2 \pi i
$$

that is in physicists terms this pictures an $U(1)$-gauge field theory over $\mathbb{R}^{2}$ with a constant nonzero curvature.

\subsection{Algebraic torus}

4.1.1 We think of elements of $\mathbb{C}^{*} \times S^{1}$ as pairs $\langle z, \exp i s\rangle$, where $z=$ $\exp (i x+y) \in \mathbb{C}^{*} x, y, s \in \mathbb{R}$.

The action of $H(\mathbb{Z})$ on $\mathbb{C}^{*} \times S^{1}$, can be given, following (21) by

$$
\begin{aligned}
& \mathbf{u}\langle\exp (i x+y), \exp i s\rangle=\langle\exp (i x+i a+y), \exp i(s+a y)\rangle \\
& \mathbf{v}\langle\exp i x+y, \exp i s\rangle=\langle\exp (i x+y+b), \exp i s\rangle
\end{aligned}
$$

The action by $\mathbf{v}$ is well-defined since it simply takes the pair $\langle z, t\rangle$ to $\left\langle e^{b} z, t\right\rangle$.

To calculate $\mathbf{u}\langle z, t\rangle$ one first takes

$$
\ln z=i x+y+2 \pi i n=i(x+2 \pi n)+y, \quad n \in \mathbb{Z} .
$$

This recovers $y$ uniquely and so $\mathbf{u}$ is well-defined.

The corresponding discrete version will be

$$
\begin{gathered}
\langle k, l, m\rangle\left\langle\exp (2 \pi i x+y), \exp 2 \pi i \frac{q}{N}\right\rangle= \\
=\left\langle\exp \left(2 \pi i\left(x+\frac{k}{N}\right)+y+\frac{\ell}{N}\right), \exp 2 \pi i \frac{q+k[N y]+m}{N}\right\rangle
\end{gathered}
$$

This is a group action, by the same calculation as in 4.0 .8

In this discrete version $t=\left\langle\exp (2 \pi i x+y)\right.$, $\exp 2 \pi i \frac{q}{N}$ is an element of $T_{N}$ and correspondingly $\mathbf{p}(t)=\exp (2 \pi i x+y)$. The $a$ and $b$ of 3.2 will be $e^{\frac{2 \pi i}{N}}$ and $e^{\frac{1}{N}}$ correspondingly.

Theorem 4 The structure on $\mathbb{C}^{*} \times\left\{\exp \frac{2 \pi i \mathbb{Z}}{N}\right\}$ in the language of 3.2 described by 4.1 .1 is isomorphic to the example $\mathrm{T}_{N}$ of [3.2 with $\mathrm{F}=\mathbb{C}$.

We want to calculate the covariant derivative following the method of 4.0.10. We use similar notation for the infinitesimal action

$$
d x=\left\{\frac{1}{N}: N \in \mathbb{N}\right\}=d y,
$$

the infinitesimal corresponding to the sequence. But the actual cooridinates on $\mathbb{C}^{*}$ are

$$
z^{1}=e^{2 \pi i x} \text { and } z^{2}=e^{y},
$$

SO

$$
d z^{1}=2 \pi i z^{1} d x, \quad d z^{2}=z^{2} d y
$$

Now for

$$
\psi: z \mapsto\left\langle x, y, e^{2 \pi i s(z)}\right\rangle
$$


the difference between the shift $d z^{1}$ and the parallel transport along the same shift will be, by the same formulas as in 4.0.10.

$$
\exp 2 \pi i(s+d s)-\exp 2 \pi i(s+y d x) .
$$

This is equal to

$$
\begin{aligned}
& \left(\frac{d \exp 2 \pi i s}{d x}-2 \pi i y \exp 2 \pi i s\right) d x= \\
& \left(\frac{d \exp 2 \pi i s}{d z^{1}}-\frac{\ln z^{2}}{z^{1}} \exp 2 \pi i s\right) d z^{1}
\end{aligned}
$$

which gives the covariant derivative along $z^{1}$

$$
\nabla_{z^{1}} \psi=\frac{d}{d z^{1}} \psi-\frac{\ln z^{2}}{z^{1}} \psi
$$

Similarly, $\nabla_{z^{2}}$ the covariant derivative along $z^{2}$ is just $\frac{d}{d z^{2}} \psi$, the second term zero.

The curvature of the connection is

$$
\left[\nabla_{z^{1}}, \nabla_{z^{2}}\right]=\frac{1}{z^{1} z^{2}}
$$

which is a nonconstant curvature (note also that $z^{1} z^{2}=\exp (2 \pi i x+y)$ does not vanish on $\mathbb{C}^{*}$ ).

\section{References}

[DFN] B.A. Dubrovin, A.T. Fomenko, S.P. Novikov, Modern geometry: methods and applications, New York; London: Springer-Verlag, 1990

[HZ] E.Hrushovski and B.Zilber, Zariski Geometries. Journal of AMS, $9(1996), 1-56$

[S] G. Svetlichny, Preparation for Gauge Theory arXiv:math-ph/9902027v1

[Z0] B.Zilber, Lecture notes on Zariski structures, 1994-2006, web-page

[Z1] B.Zilber, A class of quantum Zariski geometries, to appear in Proc. Newton Institute 2005 Programme. 